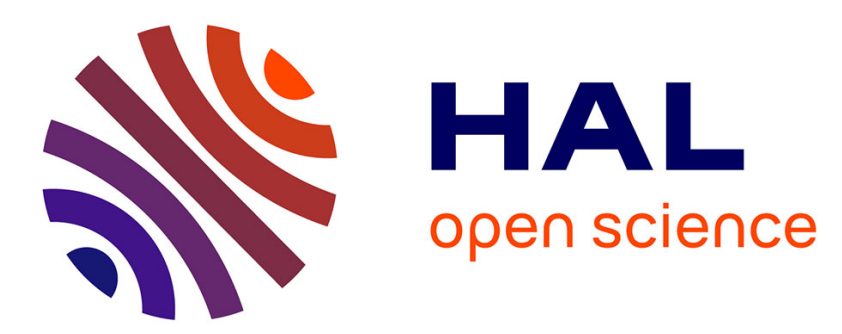

\title{
A passive scalar convective-diffusive subrange for low Prandtl numbers in isotropic turbulence
}

\author{
Antoine Briard, Thomas Gomez
}

\section{To cite this version:}

Antoine Briard, Thomas Gomez. A passive scalar convective-diffusive subrange for low Prandtl numbers in isotropic turbulence. Physical Review E : Statistical, Nonlinear, and Soft Matter Physics, 2015, $91((\mathrm{R}))$, pp.011001. 10.1103/PhysRevE.91.011001 . hal-01429650

\section{HAL Id: hal-01429650 \\ https://hal.sorbonne-universite.fr/hal-01429650}

Submitted on 9 Jan 2017

HAL is a multi-disciplinary open access archive for the deposit and dissemination of scientific research documents, whether they are published or not. The documents may come from teaching and research institutions in France or abroad, or from public or private research centers.
L'archive ouverte pluridisciplinaire HAL, est destinée au dépôt et à la diffusion de documents scientifiques de niveau recherche, publiés ou non, émanant des établissements d'enseignement et de recherche français ou étrangers, des laboratoires publics ou privés. 


\title{
A passive scalar convective-diffusive subrange for low Prandtl numbers in isotropic turbulence
}

\author{
A. Briard* and T. Gomez ${ }^{\dagger}$ \\ 1- Sorbonne Universités, UPMC Univ Paris 06, UMR 7190, \\ Institut Jean Le Rond d'Alembert, F-75005, Paris, France and \\ 2- CNRS, UMR 7190, Institut Jean Le Rond d'Alembert, F-75005, Paris, France
}

(Dated: June 2014)

\begin{abstract}
In this article, we study the behavior of a strongly diffusive passive scalar field $T$ submitted to a freely decaying, homogeneous and isotropic turbulence with Eddy-Damped Quasi-Normal Markovian simulations. We present a new subrange located between the $k^{-17 / 3}$ inertial-diffusive subrange and the Kolmogorov wavenumber $k_{\eta}$. This new subrange is generated by small scales convection linked to $k_{\eta}$ that balances diffusion effects. Thus, we build a new typical length scale $k_{C D}^{-1}$ based on convection and diffusion and give an expression for the shape of the passive scalar spectrum in this subrange $E_{T} \sim \sqrt{\operatorname{Pr}} k^{-11 / 3}$ using physical arguments. This new result unifies two different theories coming from Batchelor [G. K. Batchelor, Journal. Fluid. Mech, 5, 01 (1959)] and Chasnov [J. Chasnov et al., Phys. Fluids A, 1, 10 (1989)] and explains results previously obtained experimentally.
\end{abstract}

Vol. $91011001(R)(2015)$

Keywords: EDQNM, Isotropic turbulence, Passive scalar, Conduction, Convection

The dynamic of a passive scalar field $T$ when the Prandtl number Pr is much lower than one is a very controversial topic. There are four different theories regarding the shape of the scalar spectrum $E_{T}$. Batchelor [1](1959) proposed that $E_{T} \sim k^{-17 / 3}$ whereas Chasnov [2](1989) found $E_{T} \sim k^{-11 / 3}$ for a very rapidly stirred fluid. Moreover, Gibson [3](1968) proposed a $E_{T} \sim k^{-3}$ evolution by considering convection effects when scalar gradients are very weak or zero at small scales. Finally, Granatstein [4] (1966) established a $E_{T} \sim k^{-13 / 3}$ subrange based on experimental data in a plasma and justified it using the Heisenberg model. Thanks to EddyDamped Quasi-Normal Markovian (EDQNM) simulations $[5,6]$, a large range of Prandtl numbers $\operatorname{Pr}=\nu / a$ can be explored at high Reynolds numbers, where $\nu$ is the kinematic viscosity and $a$ the scalar diffusivity. EDQNM simulations allow to explain directly how the $k^{-13 / 3}$ could have been obtained experimentally before: this subrange was observed for fluids with $\operatorname{Pr} \in[0.1,0.01]$ and $\operatorname{Re}_{\lambda} \sim 160$ where $\operatorname{Re}_{\lambda}$ is the Reynolds number based on Taylor scale $\lambda$. However, there is no inertial-diffusive subrange for $\operatorname{Pr}=0.1$ at this Reynolds number as we can see on Fig. 3. And for $\operatorname{Pr}=10^{-2}$, the inertial-diffusive subrange is not completely established : this is probably the reason why $k^{-17 / 3}$ is not observed in experimental works [4]. In addition to this transitional Pr-state, $\operatorname{Re}_{\lambda}$ is not high enough to match with the theory (at least $\operatorname{Re}_{\lambda}=200$ is required).

In the Kolmogorov inertial subrange, $k \in\left[k_{L}, k_{\eta}\right]$ where $k_{L}$ and $k_{\eta}$ are the wavenumbers linked respectively to the integral scale and Kolmogorov scale, the kinetic spectrum $E$ evolves as

$$
E(k, t)=K_{0} k^{-5 / 3} \epsilon^{2 / 3}
$$

where $K_{0}$ is the Kolmogorov constant $(\simeq 1.5)$ and $\epsilon$ the turbulent energy dissipation rate. In the inertialconvective subrange, the scalar spectrum $E_{T}$ evolves as

$$
E_{T}(k, t)=K_{C O} \epsilon_{T} \epsilon^{-1 / 3} k^{-5 / 3}
$$

where $K_{C 0}$ is the Corrsin-Obukhov constant $(\simeq 0.66)$ and $\epsilon_{T}$ the scalar dissipation rate. The numerical values of both constants $K_{0}$ and $K_{C O}$ are recovered in our simulations. These laws for $E$ and $E_{T}$ are obtained using dimensional analysis based on physical arguments. For $\operatorname{Pr} \ll 1$ and $k \in\left[k_{C O}, k_{\eta}\right]$ where $k_{C O}=\left(\epsilon / a^{3}\right)^{1 / 4}$, there is an explicit shape for $E_{T}$ proposed by Batchelor [1]

$$
E_{T}(k, t)=\frac{K_{0}}{3} \epsilon_{T} a^{-3} \epsilon^{2 / 3} k^{-17 / 3} .
$$

Finally, the scalar spectrum $E_{T}$ satisfies the scalar Lin equation

$$
\left(\frac{\partial}{\partial t}+2 a k^{2}\right) E_{T}(k, t)=T_{T}(k, t),
$$

which is the spectral counterpart of the von Kármán Howarth equation. The non-linear scalar transfer term $T_{T}(k, t)$ is explicitly given in [6-8]. This term taking into account the triadic scalar variance transfers in wavenumber space can be accurately evaluated using EDQNM closure, as shown in $[6,9,10]$.

In this paper, the initial $\operatorname{Re}_{\lambda}$ is 23330 . We explore fluids from $\operatorname{Pr}=1$ to $\operatorname{Pr}=10^{-6}$ which can be considered as an asymptotical case of very strong diffusivity. The total simulation time is such that all quantities decrease according to Comte-Bellot-Corrsin theory [11] : this means that the decay exponents of integrated quantities such as the dissipation rate or the energy are constant. For numerical integration of equation (4), we use a logarithmic discretization in wavenumbers such that $k_{i+1}=k_{i} r(i \in \llbracket 1 ; N \rrbracket$ with typically $N=210)$ and $r=1.14369$, with a third order implicit Runge-Kutta scheme. The infrared exponents are $\sigma=\sigma_{T}=2$, so called Saffman turbulence. We present in Fig.1 the scalar spectrum $E_{T}$ for different values of Pr. First thing to notice is that the $k^{-17 / 3}$ subrange only appears for $\operatorname{Pr} \leq 10^{-3}$. Then, for $\operatorname{Pr}=10^{-6}$ the inertial-convective subrange has almost disappeared, which is expected from 


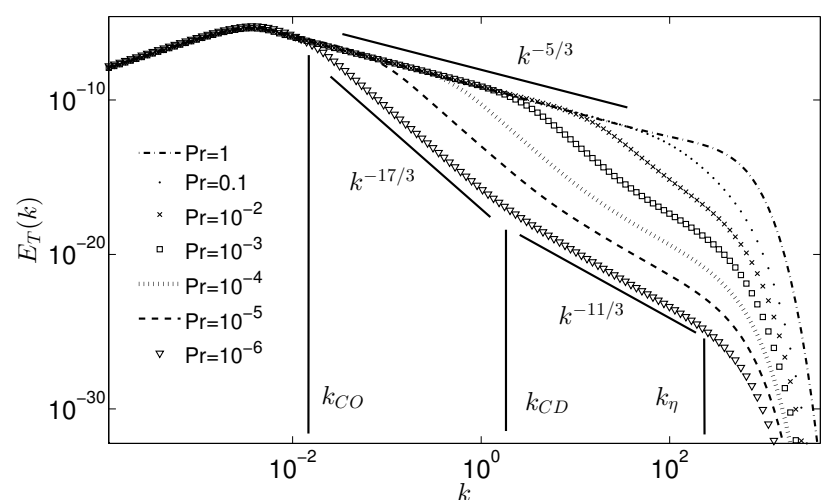

FIG. 1: Scalar spectrum $E_{T}$ for $\operatorname{Pr}=10^{-n}, n \in \llbracket 0 ; 6 \rrbracket$, with the Kolmogorov wavenumber $k_{\eta}$, the convective-diffusive wavenumber $k_{C D}$ and the Corrsin-Obukhov wavenumber $k_{C O}$.

a physical point of view, because the Péclet number $\mathrm{Pe}=\operatorname{Re}_{\lambda} \operatorname{Pr} \rightarrow 1$. We also see that there is clearly a third subrange, located between the inertial-diffusive range and the viscous-diffusive one, where $E_{T}$ decreases much slower. This effect can be understood in a simple way: as we approach the Kolmogorov wavenumber $k_{\eta}$, there is a transfer lack toward small scales and thus scalar variance $E_{T}$ accumulates.

Indeed, in the inertial-diffusive subrange, the diffusive effects are stronger than the dissipative effects near $k_{\eta}$. This new subrange is generated by convection effects that were neglected in Batchelor model [1] as mentioned by Gibson [3]. We call $k_{C D}^{-1}$ the new characteristic length scale at which this new range starts, where CD stands for convective-diffusive.

In addition to this, it is worth noting that the accumulation of $E_{T}$ in the range located just before $k_{\eta}$ is not a consequence of residual effects coming from the initial condition neither a bottleneck effect. Indeed, several initial conditions have been tried (one proposed by Pope [12], a piecewise spectra for $E$ and $E_{T}$, and one with a peak of energy at the integral scale $k_{L}$ ) and they all result in the same final behavior in the decay (i.e. matching with CBC decay exponents). Finally, bottleneck effect is not responsible for this new subrange because the simulations show that this range grows as $\sqrt{\operatorname{Pr}}$ when the Prandtl number decreases. This regular behavior does not correspond to a bottleneck effect, and this variation will be shown analytically later.

So, EDQNM simulations show three different subranges between $k_{L}$ and $k_{\eta}$ : the first one, for $k \in\left[k_{L}, k_{C O}\right]$, is the $k^{-5 / 3}$ spectrum, dominated by large scales convection effects and called the inertial-convective subrange (ICS). The designation "inertial" comes from the cascade of kinetic energy. Then, for $k \in\left[k_{C O}, k_{C D}\right]$, we have the $k^{-17 / 3}$ spectrum, called the inertial-diffusive subrange (IDS). Here, diffusive effects largely dominate and the convection by large scales is negligible. Finally, for $k \in\left[k_{C D}, k_{\eta}\right]$, as explained by Gibson [3], small scalar gradients generate non-negligible convective effects. And this time, convection comes from small scales and more precisely from the Kolmogorov wavenumber $k_{\eta}$. There, the kinetic field creates small scalar fluctuations that balance diffusion of the IDS. Thus, this new range will be referred as the inertial-balanced subrange (IBS), where "balanced" stands for an equilibrium between diffusion and convection by small scales. It will be shown that in the IBS, the scalar spectrum evolves in $k^{-11 / 3}$. One may note that the exponent $-11 / 3$ is the average between the $-5 / 3$ convection and the $-17 / 3$ diffusion.

The lack of transfer toward small scales is illustrated by plotting the budget term $k T_{T}$ in Fig.2. This quantity, often investigated [5-7], has the same dimension as the scalar flux $\Pi_{T}$ and emphasizes the role of the small scales. We can equivalenly look at $\Pi_{T}$ and $k T_{T}$ as shown by writing the following relation

$$
\Pi_{T}(k, t)=-\int_{0}^{k} T_{T}(p, t) d p=-\int_{0}^{k} p T_{T}(p, t) d(\ln p) .
$$

Figure 2 shows that the flux is conservative (areas above and below $k T_{T}=0$ are equal) and that the transfer decreases strongly in the IDS, becoming very weak in the IBS. Therefore convection of the scalar field by large scales in the IBS is not dominant. In other words, the diffusion and convection by small scales are the main phenomena which drive the scalar dynamics in the IBS range.

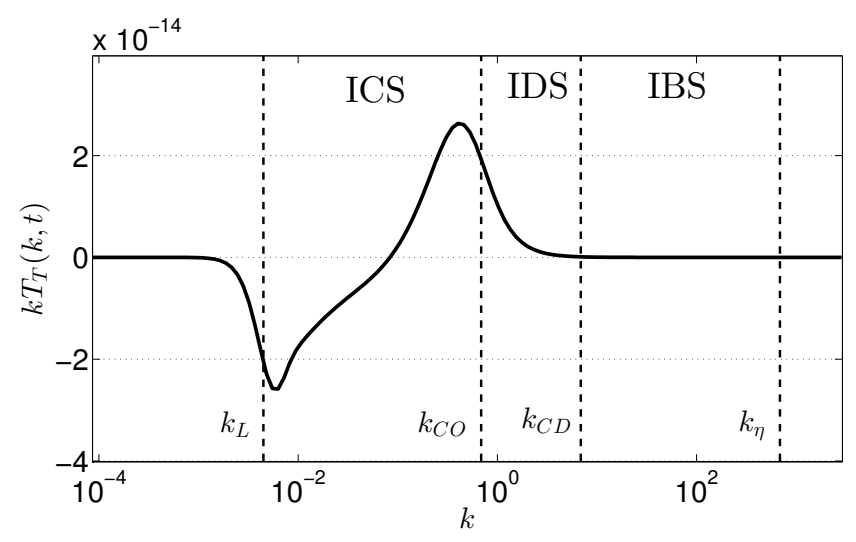

FIG. 2: Budget term $k T_{T}(k, t)$ for Pr $=10^{-4}$, with the Kolmogorov wavenumber $k_{\eta}$, the convective-diffusive wavenumber $k_{C D}$, the Corrsin-Obukhov wavenumber $k_{C O}$, and the integral wavenumber $k_{L}$.

Now, let's consider $\log _{10} E_{T} / \log _{10} k$ in Fig.3 in order to determine numerically the algebraic exponent of the IBS. It is clear that the IBS grows in size with the IDS. We obtain a $k^{-\beta}$ range for $\operatorname{Pr} \leq 10^{-4}$ where $\beta=3.55$ is obtained by a least square fit. It is also important to emphasize that in Fig. 3 the dashed line $\operatorname{Pr}=10^{-2}$ corresponds to the parameters commonly used in experiments $[4,13]$. Clearly, the exponent is approximately -4.8 , closer to $-13 / 3$ than $-17 / 3$ : as said before, it corresponds to a transitional state where Pr is not small 


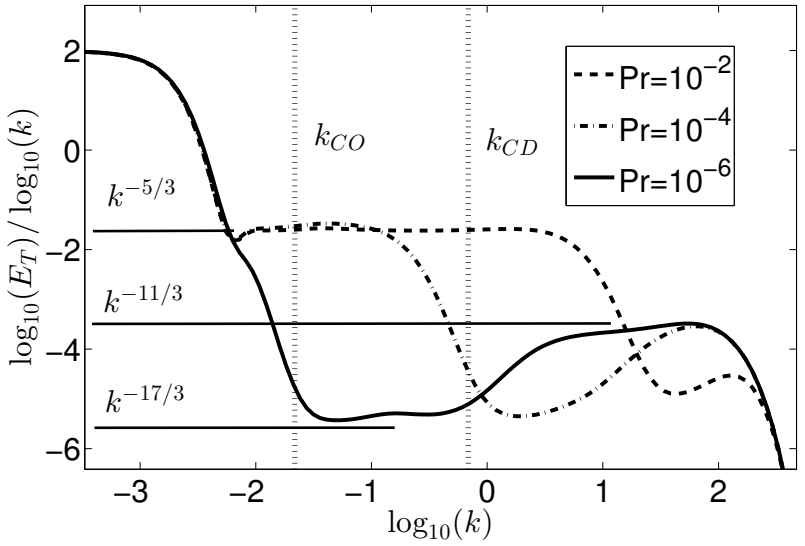

FIG. 3: $E_{T}$ exponent $\log _{10} E_{T} / \log _{10} k$ for $\operatorname{Pr}=10^{-n}, n \in$ $\llbracket 2 ; 4 ; 6 \rrbracket$ with the convective-diffusive wavenumber $k_{C D}$ and the Corrsin-Obukhov wavenumber $k_{C O}$.

enough to fully generate the IDS. In order to highlight the relevance of the new characteristic wavenumber $k_{C D}$, we plot in Fig. 3 the quantity $\log _{10} E_{T} / \log _{10} k$ and the other characteristic wavenumbers for $\operatorname{Pr}=10^{-6}$. The IDS is clearly located between $k_{C O}$ and $k_{C D}$ and the IBS after $k_{C D}$.

Let us now take a closer look at $k_{C D}$ and explicit it. As we have a competition between diffusion and convection mechanisms in the IBS, we build a length scale taking into account these two effects. To this end, we consider a characteristic diffusion time $t^{*}=k_{C O}^{-2} / a$ based on Corrsin-Obukhov wavenumber and the diffusivity. Then, we build the convective length scale using Kolmogorov characteristic velocity $u_{\eta}=(\nu \epsilon)^{1 / 4}$ and $t^{*}$. Finally, we have

$$
k_{C D}^{-1}=t^{*} u_{\eta}=\sqrt{a}\left(\frac{\nu}{\epsilon}\right)^{1 / 4} .
$$

Straightforwardly, we can then show that $k_{C D}$ verifies

$$
k_{C D}=\sqrt{\operatorname{Pr}} k_{\eta} \text { with } \operatorname{Pr} \ll 1 .
$$

One can note that this is the same relation that we have for the Batchelor wavenumber $k_{B}$ in the case $\operatorname{Pr} \gg 1$. This clearly underlines that convection effects are at the origin of the $k^{-11 / 3}$ IBS. The relevance of this expression (6) for $k_{C D}$ is illustrated in Fig.1 and 3.

In these two figures, we saw that $E_{T} \sim k^{-17 / 3}$ in the IDS $\left[k_{C O} ; k_{C D}\right]$ and then, after $k_{C D}, E_{T} \sim k^{-11 / 3}$ in the IBS $\left[k_{C D} ; k_{\eta}\right]$. This $k^{-11 / 3}$ inertial-balanced subrange (IBS) can be seen as the reconciliation of Batchelor and Chasnov theories. While Batchelor claims that for a strongly diffusive passive scalar, $E_{T}$ follows a $k^{-17 / 3}$, Chasnov [2] predicts a $k^{-11 / 3}$ law in particular conditions where the fluid is rapidly stirred. What we observe here is that the two subranges coexist when the Reynolds and Prandtl numbers are respectively large enough and small enough, and that we can find physical and theoretical arguments to explain it. Firstly, let's introduce an eddy conductivity $a_{T}$ as Chasnov [2] and Batchelor [1] did

$$
a_{T}=\int_{k}^{\infty} \frac{2 E}{3 n_{c}(k)} d k
$$

where $n_{c}^{-1}$ is a correlation time that can be seen as a characteristic time depending on the considered subrange. Using the eddy conductivity $a_{T}$, we can write the scalar dissipation $\epsilon_{T}$ as

$$
\epsilon_{T}=2\left(a+a_{T}\right) \int_{0}^{k} k^{2} E_{T} d k .
$$

The integral from 0 to $k$ takes into account the main contribution of dissipation as $k \gg 1$. The influence of small scales dynamics on $\epsilon_{T}$ is modeled by the eddy conductivity $a_{T}$. As a consequence, we can assume as Chasnov [2] did, that $d \epsilon_{T} / d k=0$. If we derive (8) with respect to $k$ and we consider that $E(k \rightarrow \infty)=0$ we find

$$
E_{T}(k, t)=\frac{K_{0}}{3} \epsilon_{T} \epsilon^{2 / 3} k^{-11 / 3}\left(a+a_{T}\right)^{-2} n_{c}^{-1} .
$$

In Batchelor's paper [1], $a_{T}$ is introduced differently. The characteristic time $n_{c}^{-1}$ appears to be $\left(a k^{2}\right)^{-1}$ which is a diffusion time. This makes sense when diffusion dominates in the $k^{-17 / 3}$ subrange. With (9), it is obvious that if $n_{c}$ does not depend on $k$, then $E_{T}$ will follow a $k^{-11 / 3}$ law. Chasnov says that for a rapidly stirred fluid - meaning that there are fluctuations at all scales - the correlation velocity-time tends to 0 and thus $n_{c}$ is constant. A physical and general interpretation of a constant $n_{c}$ could be the following one: in the IDS, the characteristic time $\left(a k^{2}\right)^{-1}$ decreases at small scales because the fluctuations produced by the kinetic field become weaker when we approach the Kolmogorov wavenumber $k_{\eta}$. At a certain point, when $k \geq k_{C D}$, convective effects play a non-negligible role and thus create a balance between convection and diffusion. And so $n_{c}^{-1}$ becomes constant. We have now to determine the value of this plateau. We have two candidates for the characteristic time, the Kolmogorov time scale

$$
\tau_{\eta}=\sqrt{\frac{\nu}{\epsilon}}
$$

and the characteristic time based on the Kolmogorov velocity scale $u_{\eta}$ and $k_{C D}$

$$
\tau_{C D}=\left(u_{\eta} k_{C D}\right)^{-1}=\sqrt{\frac{a}{\epsilon}}=\tau_{\eta} \operatorname{Pr}^{-1 / 2} .
$$

If we use the common assumption made by Chasnov, and verified numerically, that $a \gg a_{T}$, we can write (9) differently

$$
n_{c}^{-1}=\frac{E_{T}}{E} \frac{3}{\epsilon_{T}} k^{2} a^{2} .
$$

In Fig. 4 we clearly see that in the IBS, for $k \geq k_{C D}$, $n_{c}^{-1}$ becomes constant. In other words, we find the result 
predicted by Chasnov but in a more general case: indeed, we did not have to consider the case of a rapidly stirred fluid. The other point of interest is that the constant reached by $n_{c}^{-1}$ is the Kolmogorov time scale $\tau_{\eta}$. This result is consistent with the fact that the characteristic time of the convection is given by the Kolmogorov time scale when we approach Kolmogorov wavenumber $k_{\eta}$ : the IBS is generated by convection effects coming from small scales, as said before. Moreover, one can observe that in the inertial-diffusive subrange, $n_{c}^{-1}$ is proportional to $k^{-2}$ which is what was predicted by Batchelor [1] when he introduced the eddy conductivity $a_{T}$.

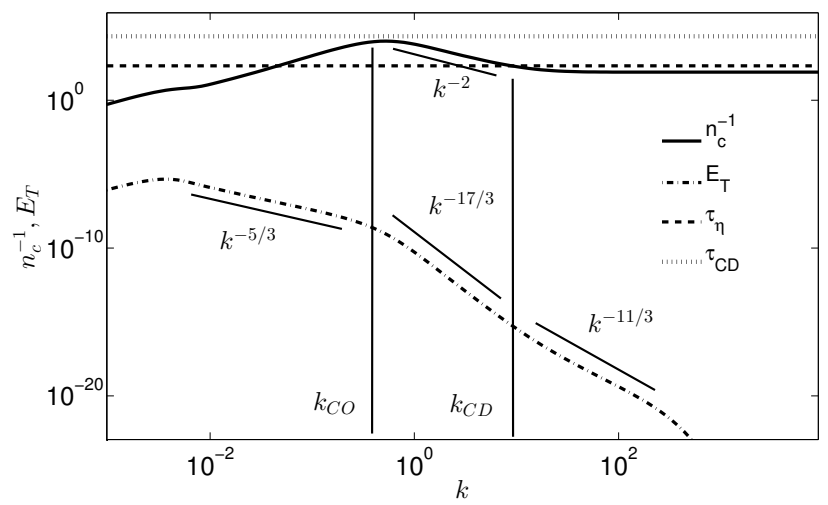

FIG. 4: Characteristic time $n_{c}^{-1}$ and scalar spectrum $E_{T}$, with the convective-diffusive wavenumber $k_{C D}$, the Kolmogorov time $\tau_{\eta}$ and the convective-diffusive time $\tau_{C D}$ for $\operatorname{Pr}=10^{-4}$.

Now that $n_{c}$ is available, we can write the scalar spectrum in the inertial-balanced subrange as

$$
E_{T}(k, t)=\frac{K_{0}}{3} \epsilon_{T} \epsilon^{1 / 6} \sqrt{\operatorname{Pr}} a^{-3 / 2} k^{-11 / 3} .
$$

\begin{tabular}{|c|c|c|}
\hline $\operatorname{Pr}$ & $\frac{\sqrt{\mathrm{Pr}}}{2}$ & Compensated $E_{T}$ \\
\hline $10^{-4}$ & 0.005 & 0.0053 \\
\hline $10^{-5}$ & 0.0016 & 0.0017 \\
\hline $10^{-6}$ & 0.0005 & 0.00053 \\
\hline
\end{tabular}

TABLE I: Variation in Pr of the compensated scalar spectrum $E_{T} \frac{3}{K_{0}} \epsilon_{T}^{-1} \epsilon^{-1 / 6} a^{3 / 2} k^{11 / 3}$ for $\operatorname{Pr}=10^{-4}, 10^{-5}$ and $10^{-6}$ when the $k^{-17 / 3}$ and $k^{-11 / 3}$ subranges are completely formed.

We note that $k^{-11 / 3}$ is in good agreement with the $k^{-3.55}$ spectrum found in numerical simulations in Fig.3. Now, if we consider the compensated scalar spectrum $E_{T} \frac{3}{K_{0}} \epsilon_{T}^{-1} \epsilon^{-1 / 6} a^{3 / 2} k^{11 / 3}=\sqrt{\operatorname{Pr}}$ in the IBS $k \in$ $\left[k_{C O}, k_{C D}\right]$ spanning on almost two decades in wavenumbers from $\operatorname{Pr}=10^{-4}$ to $\operatorname{Pr}=10^{-6}$, we assess that we obtain the $\sqrt{\mathrm{Pr}}$ dependance with EDQNM simulations. The Table I shows that the compensated scalar spectrum is indeed proportional to $\sqrt{\mathrm{Pr}}$, more precisely equal to $\sqrt{\mathrm{Pr}} / 2$ within $1 \%$ error. So in addition to the $k^{-11 / 3}$ spectrum, we have assessed the $\sqrt{\mathrm{Pr}}$ behavior. Moreover, we can also verify the hypothesis $a_{T} / a \ll 1$ by evaluating the eddy conductivity at wavenumber $k_{C D}$

$$
a_{T}=K_{0} \operatorname{Pr}^{1 / 3} \epsilon^{1 / 3}
$$

The simulations show that for $\operatorname{Pr}=10^{-4}$, when both the $k^{-17 / 3}$ and $k^{-11 / 3}$ ranges are completely formed, one have $a_{T} / a \sim 10^{-2}$, and this ratio decreases with Pr.

In conclusion, we have shown that the both theories of Chasnov and Batchelor can be merged into a single one. Thanks to EDQNM, a large range of $\operatorname{Pr}$ and $\operatorname{Re}_{\lambda}$ numbers has been explored and our theory has been assessed. We recall that the two main results are the following ones. Firstly, for a strongly diffusive scalar, $\operatorname{Pr} \ll 1$, a new $k^{-11 / 3}$ spectrum appears in the range $\left[k_{C D}, k_{\eta}\right]$ where $k_{C D}=\sqrt{\operatorname{Pr}} k_{\eta}$ is a new characteristic wavenumber based on diffusion and convection. This new subrange that we called the inertial-balanced range, is generated by convection effects that balance diffusion effects of the $k^{-17 / 3}$ inertial-diffusive subrange. These convection effects come from small scalar gradients predicted by Gibson [3] and small scales eddies of order $k_{\eta}^{-1}$. This new range appears with the $k^{-17 / 3}$ spectrum when both the Reynolds and the Prandtl numbers are respectively high enough $\left(\operatorname{Re}_{\lambda} \geq 2.10^{4}\right)$ and small enough $\left(\operatorname{Pr} \leq 10^{-3}\right)$. Finally, we determined explicitly the scalar spectrum in the inertial-balanced subrange using the eddy conductivity, $E_{T} \sim \sqrt{\operatorname{Pr}} k^{-11 / 3}$. Both variations in $k^{-11 / 3}$ and $\sqrt{\mathrm{Pr}}$ are verified by EDQNM simulations. Using analytical and numerical approaches, we have brought a new general result regarding the homogeneous and isotropic turbulence for the passive scalar. This result is a further step forward in the field of the passive scalar dynamics.

* Electronic address: antoine.briard@upmc.fr

† Electronic address: thomas.gomez@upmc.fr

[1] G. K. Batchelor, Journal. Fluid. Mech Vol. 5, 01 (1959)

[2] J. Chasnov et al., Phys. Fluids Vol. 1, 10 (1989)

[3] C. H. Gibson, Phys. Fluids Vol. 11, 11 (1968)

[4] V. L. Granatstein et al., Phys. Rev. Let Vol. 16, 12 (1966)

[5] P. Sagaut and C. Cambon, Cambridge University Press (2008)

[6] M. Lesieur, Springer (2008)

[7] A. A. Townsend, Cambridge University Press (1976)

[8] G. K. Batchelor, Cambridge University Press (1959)

[9] M. Larcheveque et al., Turbulent Shear Flows II (Springer) (1980)

[10] J. R. Herring et al., Journal. Fluid. Mech Vol. 124 (1982)

[11] S. Corrsin, Journal. Aero. Science Vol. 18, 417 (1951)

[12] S. B. Pope, Cambridge University Press (2000)

[13] J. H. Rust and A. Sesonske J. Heat Mass Transfer Vol. 9 (1966) 